Radiologe 2017 · 57:615-620

DOI 10.1007/s00117-017-0274-3

Online publiziert: 12. Juli 2017

(c) Der/die Autor(en) 2017. Dieser Artikel ist eine Open-Access-Publikation.

CrossMark

\author{
F. Steinkohl' $\cdot$ A. Luger ${ }^{1} \cdot$ J. Bektic ${ }^{2} \cdot$ F. Aigner ${ }^{1}$ \\ ' Universitätsklinik für Radiologie, Medizinische Universität Innsbruck, Innsbruck, Österreich \\ ${ }^{2}$ Universitätsklinik für Urologie, Medizinische Universität Innsbruck, Innsbruck, Österreich
}

\title{
Ultraschall der Prostata
}

\section{Vom B-Bild über den multiparametrischen Ultraschall zur gezielten Biopsie}

\begin{abstract}
Das Prostatakarzinom ist das häufigste Karzinom des Mannes, deshalb kommt der Früherkennung eine besondere Bedeutung zu. Diese erfolgt durch die digital-rektale Untersuchung und durch die Bestimmung des prostataspezifischen Antigens (PSA). Erhöhte PSA-Werte stellen genauso wie ein auffälliger Tastbefund eine Indikation zur Prostatabiopsie dar.
\end{abstract}

\section{Hintergrund}

Üblicherweise erfolgt eine sogenannte systematische Biopsie (SB) mit dem transrektalen Ultraschall im B-Bild-Modus (B-TRUS). Der B-TRUS stellt die Prostata in Grauwerten dar. Er dient lediglich der Erkennung des Organs und definierter Zonen (Sextantenschema). Der Untersucher entnimmt aus diesen vorgegebenen Sextanten der Prostata (Basis, Mitte und Apex, je rechts und links) mindestens je 2 Gewebeproben. Bei der SB wird in der Regel nicht auf karzinomsuspekte Veränderungen geachtet, da im B-TRUS Prostatakarzinome nicht nur hypo-, sondern auch iso- oder hyperechogen sein können.

Der Vorteil dieses randomisierten Ansatzes liegt darin, dass keine vertieften Kenntnisse in speziellen Ultraschalltechniken wie der Dopplersonographie oder der Elastographie notwendig sind. Der Nachteil besteht darin, dass nicht aus allen Bereichen der Prostata Gewebeproben entnommen werden (z. B. weit anterior). Einerseits werden so bis zu $30 \%$ signifikanter Karzinome nicht detektiert $[1,2]$, andererseits detektiert die SB aber eine hohe Zahl insignifikan- ter Prostatatumoren [3]. Insignifikante Prostatakarzinome haben keinen Krankheitswert und benötigen keine Therapie. Eine hohe Zahl detektierter insignifikanter Karzinome (Überdiagnose) führt zu einer hohen Zahl an unnötigen Therapien (Übertherapie). Die geringe Sensitivität der SB zum Nachweis signifikanter Prostatakarzinome und die geringe Spezifität des PSA-Werts stellen in der Prostatakarzinomdiagnostik ein echtes Dilemma dar.

Moderne bildgebende Techniken haben mittlerweile bewiesen, dass sie relativ zuverlässig signifikante Prostatakarzinome nachweisen können [4]. Daher kommt der Visualisierung des Prostatakarzinoms eine wichtige Rolle zu. Neben der multiparametrischen MRT, die als Goldstandard in der Prostatabildgebung gilt, werden in einigen spezialisierten Zentren auch moderne TRUS-Techniken angewendet: Einerseits, um die Detektionsrate signifikanter Prostatakarzinome zu erhöhen, andererseits um alternative Therapiestrategien zur radikalen Prostatektomie anbieten zu können, wie beispielsweise die fokale Therapie oder Active Surveillance.

\section{Multiparametrischer TRUS (mpTRUS)}

Ähnlich der multiparametrischen MRT wird die gleichzeitige Anwendung struktureller (B-TRUS) und funktioneller TRUS-Techniken unter dem Begriff multiparametrischer TRUS (mpTRUS) zusammengefasst [5]. Auch der mpTRUS zeigt, dass eine Ergänzung des B-Bildes um funktionelle Gewebeinformationen, wie der Gewebehärte oder des
Durchblutungsmusters, zu einer erhöhten diagnostischen Genauigkeit in der Prostatakrebsdiagnostik führt [6]. Der multiparametrische Ultraschall wird nicht nur bei Prostatafragestellungen verwendet, sondern hat auch in anderen Organen (z. B. Hoden und Mamma) sein Potenzial unter Beweis stellen können.

Neben dem strukturellen B-TRUS kommen funktionelle TRUS-Techniken wie Ultraschallelastographie (USE), Doppler-TRUS (D-TRUS) und eventuell auch der kontrastmittelverstärkte TRUS (CE-TRUS) zur Anwendung. Auch weitere Techniken wie beispielsweise der 3-D-/4-D-TRUS oder Fusionstechniken haben in der Diagnostik von Prostataerkrankungen Einzug gehalten.

\section{Normalbefunde}

\section{B-Bild-Ultraschall der Prostata (B-TRUS)}

Der B-TRUS ermöglicht eine gute anatomische Darstellung der Prostata und ist daher als sonographisches Pendant der strukturellen T2-Sequenzen im MRT anzusehen. Neben der Steuerung der SB dient er auch zur Volumetrie der Prostata, zur Beurteilung der Kapsel und zur Darstellung der Gewebeverschieblichkeit der Prostata gegenüber der Rektumwand bzw. dem periprostatischen Fettgewebe. Außerdem können Samenblasen und -leiter gut erkannt werden. Dies ist für Fragestellungen in der Andrologie wichtig (z. B. Azoospermie).

Die gesunde Prostata besteht aus:

- einer bandförmigen, echoreicheren

peripheren Zone (PZ), die viel

Drüsengewebe besitzt (• Abb. 1a), 

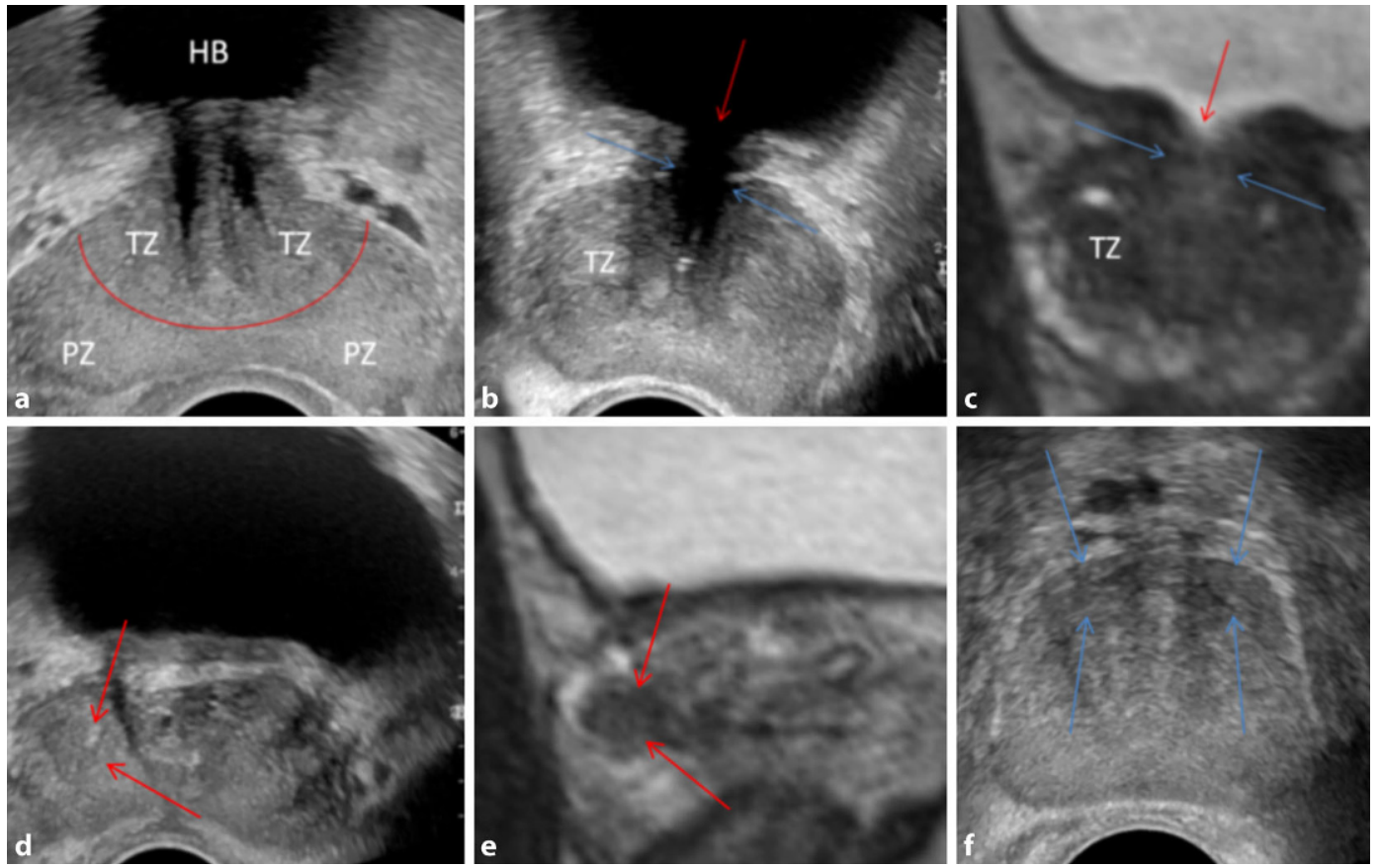

Abb. $1 \Delta$ a Bandförmig konfigurierte, echoreiche periphere Zone (PZ), die rote Linie ist die Begrenzung zwischen PZ und Transitionalzone (TZ; HB Harnblase). b Die TZzeigt sich als echoärmere, meist knotig konfigurierteZone aufHöhe der Prostatamitte mit Harnblasenhals (roterPfeil), die periurethrale Zone (blaue Pfeile) zeigt sich echoarm und tubulär konfiguriert. c Entspricht der T2-gewichteten Darstellung von $\mathbf{b}$ in der MRT. d,e Echoärmere, meist knotig bis tränentropförmig konfigurierte zentrale Zone im B-Bild (rote Pfeile in d) und in der korrespondierenden T2-gewichteten MRT (rote Pfeile in e). $\mathbf{f}$ Die blauen Pfeile markieren das sichel- bis kappenförmige, symmetrisch angeordnete und echoarme anteriore, fibromuskuläre Stroma

- einer echoärmeren, meist knotig konfigurierten Transitionalzone (TZ), mit reichlich Muskulatur und Bindegewebe (•Abb. 1b, c),

- einer echoärmeren, meist tubulär konfigurierten periurethralen Zone, welche die Harnröhre umgibt und vom Blasenhals bis zur membranösen Harnröhre erkennbar ist (- Abb. 1b, c),

- einer echoärmeren, meist knotig bis tränentropförmig konfigurierten zentralen Zone (ZZ), welche die Ductus deferentes und ejaculatorii umgibt (• Abb. 1d, e),

- einem sichel- bis kappenförmigen, symmetrisch angeordnetem, echoarmen und kaum durchbluteten anterioren, fibromuskulären Stroma (AFS), welches die Prostata nach ventral im kleinen Becken fixiert (•Abb. 1f).

\section{Ultraschallelastographie (USE)}

Ähnlich der digital-rektalen Untersuchung kann auch die USE die Gewebeelastizität erfassen, indem sie diese farblich kodiert am Ultraschallmonitor darstellt. Daher kann die USE als technischer Finger verstanden werden, wobei in den hier verwendeten Abbildungen harte und somit karzinomverdächtige Areale blau, Areale weicher oder intermediärer Gewebeelastizität rot oder grün kodiert sind [7]. Das unauffällige Elastogramm einer normgroßen, weichen Prostata eines jungen Mannes zeigt - Abb. 2a, b. Mit zunehmendem Alter hypertrophiert die innere Drüse und wird zusehends härter, sodass mithilfe der USE - ähnlich der diffusionsgewichteten MRT (DWI) - der Prostatakarzinomnachweis immer schwieriger wird (-Abb. 2c, d).

\section{Doppler-TRUS (D-TRUS)}

Die Dopplertechniken geben Aufschluss über die Durchblutung des Organs, insbesondere über die Gefäßarchitektur und den Vaskularisationsgrad. Auflösungsbedingt kommt es lediglich $\mathrm{zu}$ einer Darstellung der Makrozirkulation. Im Normalfall stellt sich die äußere Drüse im Vergleich zur inneren hypovaskulär mit einem radiärem Gefäßmuster dar (- Abb. 2e, f; [8]).

\section{Kontrastmittelverstärkter TRUS (CE-TRUS)}

Im Gegensatz zum D-TRUS können nach intravenöser Applikation von Ultraschallkontrastmittel nicht nur Informationen über die Makro-, sondern auch die Mikrozirkulation gewonnen werden. Die Durchblutung wird mit einer hohen räumlichen und zeitlichen Auflösung dargestellt. Im Normalfall ist die äußere Drüse im Vergleich zur inneren hypovaskulär und zeigt ein langsameres Anfluten sowie eine geringere Aufnahme des Kontrastmittels (- Abb. 2g).

\section{Prostatakarzinom}

\section{Äußere Drüse (periphere Zone)}

Das typische Karzinom der äußeren Drüse stellt sich echoarm und nodulär konfiguriert im B-TRUS (Verlust der drüsigen Anteile und damit Verlust der Reflexivität), hart in der USE (Zunahme der Zelldichte, Verminderung der Elastizität) sowie als umschriebene Herdbildung erhöhter Vaskularisation mit Aufhebung der normalen, radiären Gefäßarchitektur dar (• Abb. 3a-c). Verstärkte sowie rasche 
Aufnahme von Ultraschallkontrastmittel und ein rasches „wash-out" nach Erreichen des Peaks sind tumorverdächtige Zeichen im CE-TRUS (• Abb. 3d, e; [9]).

\section{Innere Drüsenanteile}

Die innere Drüse stellt für alle bildgebenden Modalitäten eine Herausforderung dar. Aufgrund ihrer histologischen $\mathrm{Zu}$ sammensetzung, der mit zunehmendem Alter auftretenden Hypertrophie und der oft ablaufenden entzündlichen Prozesse präsentiert sie sich häufig hart, diffusionsgestört und hypervaskulär. Dies sind auch die Gründe, warum die strukturellen Techniken (T2-gewichtete MRT und B-TRUS) die führenden Techniken zum Karzinomnachweis in der inneren Drüse sind. Diesem Umstand wird in der PIRADS v2 insofern Rechnung getragen, als die DWI und die kontrastmittelverstärkte MRT nur noch eine untergeordnete Rolle spielen [10].

Die klassischen Erscheinungsformen eines Karzinoms in diesen Prostataabschnitten sind im B-TRUS lineare, flächige, lentikuloforme, echoarme Veränderungen mit oder ohne „bulging“ (• Abb. 4; [11]).

\section{Prostatabiopsie}

\section{Systematische Biopsie (SB)}

Die diagnostische Genauigkeit des B-TRUS wird in der Literatur als unzureichend zur Prostatadetektion eingeschätzt [12]. Deswegen wird der B-TRUS von den Urologen im Rahmen der SB lediglich zur Erkennung jener Zonen verwendet, aus denen die randomisierten Biopsate entnommen werden sollten. Aus tief apikalen, weit anterior gelegenen Arealen sowie aus der Transitionalzone werden in der SB routinemäßig keine Gewebeproben entnommen, da die Auffassung bestand/teils noch besteht, die Mehrzahl aller relevanten Prostatakarzinome würde aus den dorsolateralen Anteilen der äußeren Drüse entstehen [13].

\section{B-TRUS-gezielte Biopsie}

Tatsächlich könnte dem B-TRUS aber eine bedeutendere Rolle in der Karzi-

Radiologe 2017·57:615-620 DOI 10.1007/s00117-017-0274-3

(c) Der/die Autor(en) 2017. Dieser Artikel ist eine Open-Access-Publikation.

F. Steinkohl · A. Luger · J. Bektic · F. Aigner

Ultraschall der Prostata. Vom B-Bild über den
multiparametrischen Ultraschall zur gezielten Biopsie

\section{Zusammenfassung}

Hintergrund. Das Prostatakarzinom ist das häufigste Karzinom des Mannes. Die Diagnose erfolgt üblicherweise durch eine systematische Prostatabiopsie. Dies ist aber ein randomisierter Ansatz, bei dem eine beträchtliche Anzahl signifikanter Karzinome nicht erfasst werden. Deshalb wurden in den letzten Jahren bildgebende Techniken stetig weiterentwickelt, die eine Visualisierung und somit eine gezielte Biopsie ermöglichen. Standardverfahren. Die systematische Biopsie ist das Standardverfahren, um Prostatakarzinome nachzuweisen. Die Qualität der Biopsie kann gesteigert werden, indem die Prostata während der Biopsie auf das Vorliegen karzinomsuspekter Veränderungen untersucht wird. Dies kann durch den multiparametrischen transrektalen Ultraschall geschehen.

\section{Ultrasonography of the prostate gland. From B-image through multiparametric ultrasound to targeted biopsy}

\section{Abstract}

Background. Prostate cancer is the most frequent cancer in men. The diagnosis is normally achieved by a systematic prostate biopsy; however, this is a randomized approach by which a substantial number of significant carcinomas go undetected. For this reason, in recent years imaging techniques have been continuously developed, which enable visualization and therefore targeted biopsies.

Standard procedure. The use of systematic biopsies is a standard procedure for the detection of prostate cancer. The quality of biopsies can be increased if the prostate is examined for the presence of suspected cancerous alterations during the biopsy. This can be carried out using multiparametric transrectral ultrasound.

Performance. Multiparametric ultrasound within the framework of a targeted biopsy
Leistungsfähigkeit. Der multiparametrische Ultraschall erhöht im Rahmen der gezielten Biopsie die Detektionsrate signifikanter Prostatakarzinome bei gleichzeitig geringerer Detektion insignifikanter Karzinome. Allerdings sind die diagnostische Zuverlässigkeit und der Evidenzlevel des mpTRUS noch nicht hoch genug, um auf eine systematische Biopsie verzichten zu können.

Bewertung. Der multiparametrische transrektale Ultraschall stellt bereits jetzt in der Hand eines geübten Untersuchers eine gute Methode zur Detektion von Prostatakarzinomen dar. Mit der fortschreitenden technischen Entwicklung der Ultraschallgeräte wird die Detektionsrate vermutlich weiter steigen.

Schlüsselwörter

Prostatakarzinom - mpTRUS - Diagnostische Zuverlässigkeit · Evidenzlevel · Detektionsrate

\begin{abstract}
increases the detection rate of significant prostate carcinomas with a simultaneous decrease in detection of insignificant carcinomas; however, the diagnostic reliability and the evidence level of multiparametric transrectal ultrasound are not yet sufficiently high to be able to replace a systematic biopsy. Conclusion. In the hands of a well-trained examiner multiparametric transrectal ultrasound represents a good method for detection of prostate carcinomas. With the progression in technical developments of ultrasound technology, the detection rate will presumably be further increased.
\end{abstract}

\section{Keywords}

Prostate cancer - Multiparametric transrectal ultrasound · Diagnostic reliability - Evidence level - Detection rate nomerkennung zukommen. Das liegt zum einen an den technischen Fortschritten der Ultraschallgeräte und zum anderen kann man durch mpTRUS-/ mpMRT-gezielte Fusionsbiopsien sehr viel zur B-Bild-Morphologie von Prosta- takarzinomen dazulernen. Daher kann man mittlerweile davon ausgehen, dass die Sensitivität zum Nachweis von Karzinomen in der äußeren Drüse über den angegebenen Werten liegt, wenn ein erfahrener Untersucher die Sonographie 

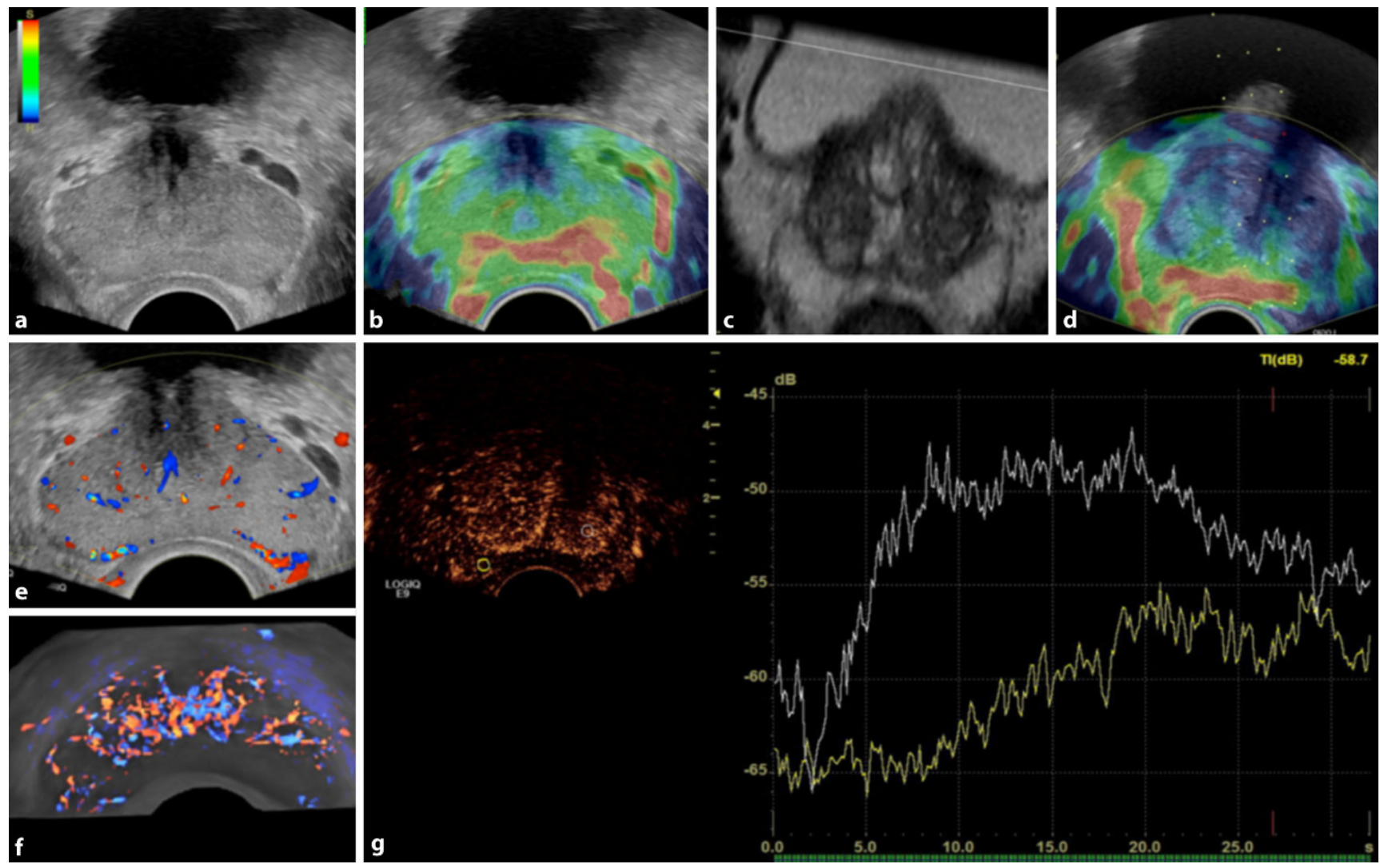

Abb. 2 ム Multiparametrischer transrektaler Ultraschall (mpTRUS) ohne Karzinomverdacht. a Normal große Prostata im B-Bild mit b hoher bis intermediärer Elastizität im korrespondierenden Elastogramm (grün bis rot kodiert; beachte die normale periurethrale Verhärtung - blau kodiert). Das T2-gewichtete MRT (c) und das korrespondierende Elastogramm (d) zeigen eine hypertrophierte innere Drüse, die diffus verhärtet ist (blau kodiert). e,f Im Vergleich zur Transitionalzone (TZ) hypovaskuläre periphere Zone (PZ) in der Dopplersonographie. $\mathbf{g}$ Graphische Darstellung der Kontrastmittel(KM)-Dynamik mit langsamerer und geringerer KM-Aufnahme der PZ (grüne Kurve) im Vergleich zur TZ (weiße Kurve)
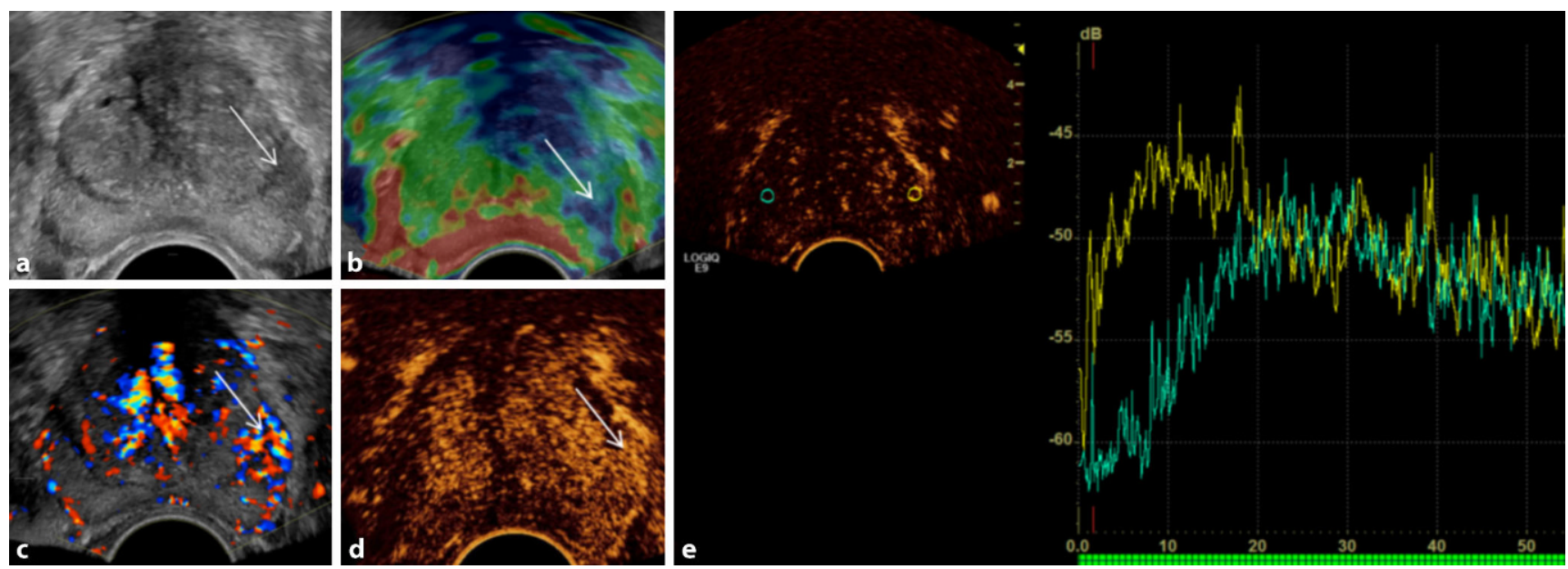

Abb. 3 - Multiparametrischer transrektaler Ultraschall (mpTRUS) mit Karzinom der peripheren Zone (PZ) links anterolateral in der Prostatamitte (Pfeile in a-d und gelbe Kurve in e). Das Karzinomareal zeigt sich im B-Bild nodulär und hypoechogen (a), verhärtet in der Elastographie (blau kodiert, b), hypervaskulär im Doppler-Trus (D-TRUS) mit gestörter Gefäßarchitektur (c), als Fokus vermehrter und umschriebener Kontrastmittel(KM)-Aufnahme im kontrastmittelverstärkten(CE)-TRUS (d) sowie mit pathologischer KM-Kurve in der Zeit-Intensitäts-Analyse vom Wash-out-Typ (e) 

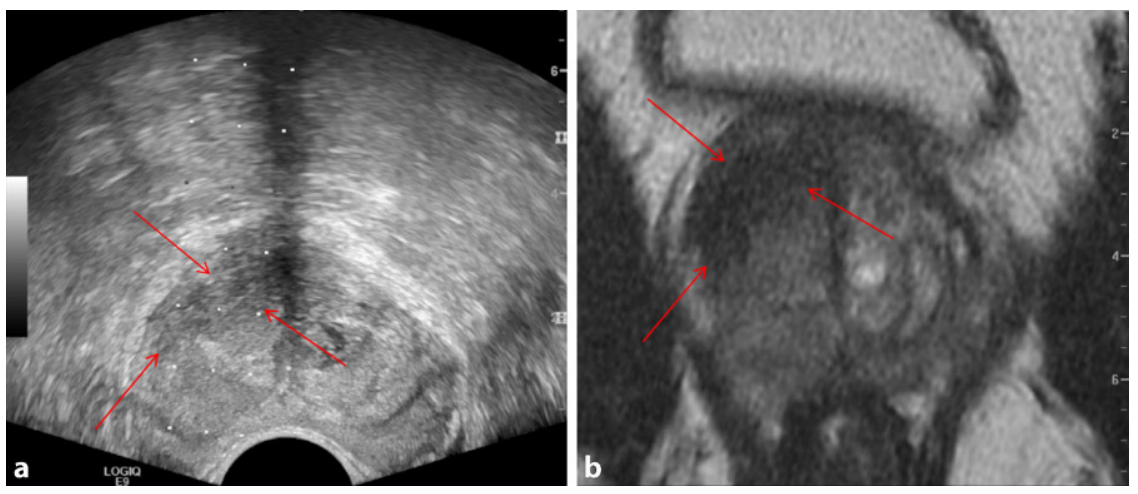

Abb. 4 \ Großes und weit anterior gelegenes Prostatakarzinom (rotePfeile) mit deutlicher Signalabsenkung und unscharfer Berandung in der T2-gewichteten MRT (b); das im Fusionsmodus generierte B-Bild zeigt den Herd stark hypoechogen, ebenfalls mit unscharfer Berandung (a)
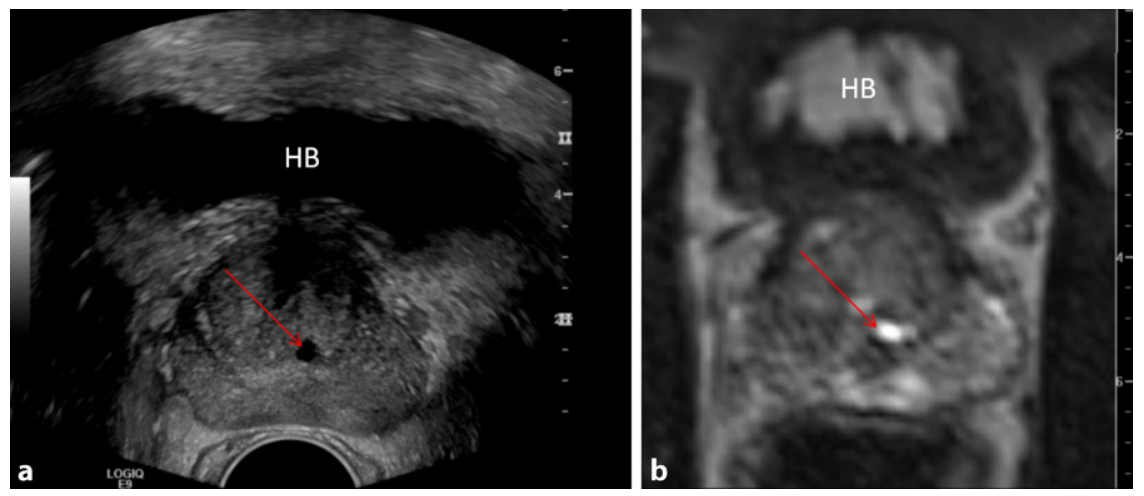

Abb. 5 ॥ Guter Schichtabgleich zwischen Ultraschall (a) und MRT (b) auf Höhe einer Zyste (roter Pfeil), die als Landmarke dient. HB Harnblase

\section{Tab. 1 Vor-und Nachteile des mpTRUS}

\begin{tabular}{ll}
\hline Vorteile & Nachteile \\
\hline $\begin{array}{l}\text { Kann von Urologen und Radiologen durchge- } \\
\text { führt werden }\end{array}$ & Untersucherabhängigkeit (Freihandtechnik) \\
\hline $\begin{array}{l}\text { Prostatabiopsie unter Echtzeitbedingungen } \\
\text { Steigert die Detektionsrate klinisch signifikan- }\end{array}$ & $\begin{array}{l}\text { Probleme in der Transitionszone und bei weit } \\
\text { anterior lokalisierten Karzinomen in großen } \\
\text { Organen }\end{array}$ \\
$\begin{array}{l}\text { Für den niedergelassenen Bereich zurzeit noch } \\
\text { zu teuer in der Anschaffung (v. a. USE und } \\
\text { CE-TRUS, Hard- und Software) }\end{array}$ \\
$\begin{array}{l}\text { Senkt die Detektionsrate klinisch insignifikan- } \\
\text { ter Prostatakarzinome }\end{array}$ & $\begin{array}{l}\text { Niedriger Evidenzlevel bei Fehlen von Multi- } \\
\text { centerstudien }\end{array}$ \\
$\begin{array}{l}\text { Potenzial zur flächendeckenden Implemen- } \\
\text { tierung (in jeder urologischen Praxis steht ein } \\
\text { Ultraschallgerät) }\end{array}$ & $\begin{array}{l}\text { Schallauslöschung bei Verkalkungen } \\
\text { (• Abb. 6) }\end{array}$ \\
\hline
\end{tabular}

durchführt. Erste Studien bestätigen diese Annahme: Eine Arbeitsgruppe aus den Niederlanden berichtete über eine $62 \%$ ige Darstellbarkeit aller als PIRADS 5 eingestufter MRT-Veränderungen im B-Bild (• Abb. 4; [14, 15]).

\section{MpTRUS-gezielte Biopsie}

Eine Analyse von 1024 Männern, die sich einer kombinierten Prostatabiopsie bestehend aus einer SB mit 10 Stanzen und einer USE-gezielten Biopsie mit 4Stanzen

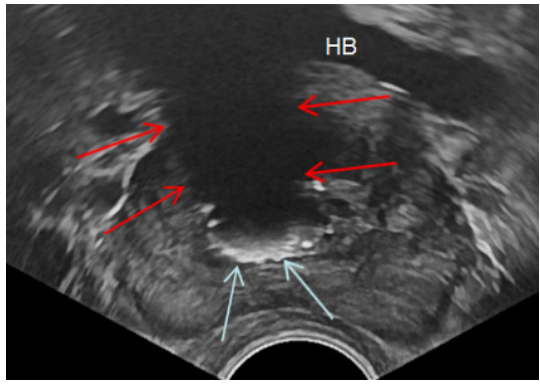

Abb. 6 \ Prostataverkalkungen (weiße Pfeile), dadurch verursachte dorsale Schallauslöschung (rote Pfeile) mit nicht beurteilbarem Bereich. HB Harnblase

$18,3 \%$ in der Karzinomdetektion führte (• Abb. 5; [16]). Von diesen zusätzlich und allein durch die USE entdeckten Karzinomen war immerhin fast die Hälfte klinisch signifikant, die der SB entgangen waren. Wenn man zum strukturellen B-Bild und zur USE noch Informationen über die Perfusion hinzufügt, reduziert man einerseits die falsch-positiven Raten [17] und erhöht andererseits die Detektionsrate, die bezogen auf die äußere Drüse ähnlich jener der mpMRT-gezielten Biopsie liegt [9].

\section{MpTRUS-/mpMRT-gezielte Fusionsbiopsien}

Bei dieser Technik wird ein MRT-Datensatz der Prostata in das Ultraschallgerät eingespielt, der dann nach Schichtabgleich und Koregistrierung simultan mit dem Echtzeitultraschall mitbewegt werden kann. Diese Art der gezielten Prostatabiopsie ist aus folgenden Gründen interessant $[4,18]$ :

- man kann außerhalb des MR-

Scanners ultraschallgeführt gezielt

Herdbefunde biopsieren, die in der mpMRT karzinomsuspekt sind,

- es werden 2 moderne bildgebende

Verfahren, nämlich der mpTRUS und die mpMRT zusammengeführt

(- Abb. 6), unterzogen, zeigte, dass die Einbindung von Informationen über die Gewebehärte $\mathrm{zu}$ einem diagnostischen Mehrwert von
- die Bildinterpretation wird durch den TRUS/MRT-Abgleich verbessert.

\section{Outcomegezielte Biopsiestrategien}

Für gezielte Biopsiestrategien gilt, dass sie im Vergleich zur SB mehr klinisch signifikante Karzinome mit weniger Stanzen 
und auch weniger indolente Karzinome nachweisen [11]. Trotzdem scheint die Sensitivität der alleinigen gezielten Biopsie noch nicht hoch genug zu sein, sodass die Empfehlung besteht, an eine gezielte Biopsie eine SB anzuschließen [19]. In - Tab. 1 werden Vor- und Nachteile des mpTRUS zusammengefasst.

\section{Fazit für die Praxis}

- Die simultane Anwendung struktureller (B-Bild-Ultraschall) und funktioneller (Dopplerultraschall und Ultraschallelastographie, ggf. Kontrastmittelultraschall) wird als mpTRUS bezeichnet.

- Durch den mpTRUS wird die diagnostische Qualität der Prostatabiopsie erhöht.

- Der mpTRUS kann zurzeit die systematische Biopsie nicht ersetzen (niedriger Evidenzlevel), sodass er noch in Kombination mit der systematischen Prostatabiopsie durchgeführt wird.

- Da der transrektale Ultraschall häufig von Urologen durchgeführt wird und in fast jeder urologischen Praxis ein Ultraschallgerät steht, hat der mpTRUS das Potenzial, flächendeckend angeboten zu werden.

\section{Korrespondenzadresse}

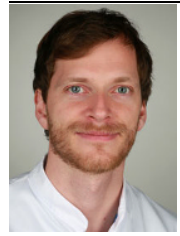

\section{Dr. F. Steinkoh}

Universitätsklinik für Radiologie, Medizinische Universität Innsbruck Anichstraße 35, 6020 Innsbruck, Österreich fabian.steinkohl@tirolkliniken.at

Acknowledgements. Open access funding provided by University of Innsbruck and Medical University of Innsbruck.

\section{Einhaltung ethischer Richtlinien}

Interessenkonflikt. F. Steinkohl, A. Luger, J. Bektic und F. Aigner geben an, dass kein Interessenkonflikt besteht.

Dieser Beitrag beinhaltet keine von den Autoren durchgeführten Studien an Menschen oder Tieren.
Open Access. Dieser Artikel wird unter der Creative Commons Namensnennung 4.0 International Lizenz (http://creativecommons.org/licenses/by/4.0/deed. de) veröffentlicht, welche die Nutzung, Vervielfältigung, Bearbeitung, Verbreitung und Wiedergabe in jeglichem Medium und Format erlaubt, sofern Sie den/die ursprünglichen Autor(en) und die Quelle ordnungsgemäßnennen, einen Link zur Creative Commons Lizenz beifügen und angeben, ob Änderungen vorgenommen wurden.

\section{Literatur}

1. Serefoglu EC, Altinova S, Ugras NS et al (2013) How reliable is 12 -core prostate biopsy procedure in the detection of prostate cancer? Can Urol Assoc J 7:E293-E298

2. Peltier A, Aoun F, El-Khoury F et al (2013) 3D versus 2D systematic transrectal ultrasound-guided prostate biopsy: higher cancer detection rate in clinical practice. Prostate Cancer 2013:783243

3. Bjurlin MA, Taneja SS (2014) Standards for prostate biopsy. Curr Opin Urol 24:155-161

4. Robertson NL, Emberton M, Moore CM (2013) MRItargeted prostate biopsy: a review of technique and results. Nat Rev Urol 10:589-597

5. Sidhu PS (2015) Multiparametric Ultrasound (MPUS) imaging: terminology describing the many aspects of ultrasonography. Ultraschall Med 36:315-317

6. Postema A, Idzenga T, Mischi $M$ et al (2015) Ultrasound modalities and quantification: developments of multiparametric ultrasonography, a new modality to detect, localize and target prostatic tumors. Curr Opin Urol 25:191-197

7. Salomon G, Schiffmann J (2014) Real-time elastography for the detection of prostate cancer. Curr Urol Rep 15:392

8. Aigner $F$, Mitterberger $M$, Rehder $P$ et al (2010) Status of transrectal ultrasound imaging of the prostate. JEndourol 24:685-691

9. Aigner F, Schafer G, Steiner E et al (2012) Value of enhanced transrectal ultrasound targeted biopsy for prostate cancer diagnosis: a retrospective data analysis. World JUrol 30:341-346

10. Weinreb JC, Barentsz JO, Choyke PL et al (2016) PI-RADS Prostate Imaging - Reporting and Data System: 2015, Version 2. Eur Urol 69:16-40

11. Aigner F, Pallwein-Prettner L (2016) Prostata: Multimodale Bildgebung, 1. Aufl. Breitenseher, Horn, S 135

12. Trabulsi EJ, Sackett D, Gomella LG et al (2010) Enhanced transrectal ultrasound modalities in the diagnosis of prostate cancer. Urology 76:1025-1033

13. Haffner J, Potiron E, Bouye S et al (2009) Peripheral zone prostate cancers: location and intraprostatic patterns of spread at histopathology. Prostate 69:276-282

14. Ukimura O, Marien A, Palmer S et al (2015) Trans-rectal ultrasound visibility of prostate lesions identified by magnetic resonance imaging increases accuracy of image-fusion targeted biopsies. World JUrol 33:1669-1676

15. van de Ven WJ, Sedelaar JP, van der Leest MM et al (2016) Visibility of prostate cancer on transrectal ultrasound during fusion with multiparametric magnetic resonance imaging for biopsy. Clin Imaging 40:745-750

16. Salomon G, Drews N, Autier P et al (2014) Incremental detection rate of prostate cancer by real-time elastography targeted biopsies in combination with a conventional 10-core biopsy in 1024 consecutive patients. BJU Int 113:548-553

17. Brock M, Eggert T, Palisaar RJ et al (2013) Multiparametric ultrasound of the prostate: adding contrast enhanced ultrasound to realtime elastography to detect histopathologically confirmed cancer.J Urol 189:93-98

18. Maxeiner A,Stephan C, DurmusTetal (2015) Added value of multiparametric ultrasonography in magneticresonanceimaging and ultrasonography fusion-guided biopsy of the prostate in patients with suspicion for prostate cancer. Urology 86:108-114

19. Hansen NL, Kesch C, Barrett T et al (2016) Multicentre evaluation of targeted and systematic biopsiesusing magneticresonanceandultrasound image-fusion guided transperineal prostate biopsy in patients with a previous negative biopsy. BJUInt. doi:10.1111/bju.13711 